Revue d'histoire de l'Amérique française

W REVUE D.HISTOIRE DE L'AMÉRIQUE FRANÇAISE

\title{
Olson, Sherry et Patricia Thornton, Peopling the North American City, Montreal 1840-1900 (Montréal et Kingston, McGill-Queen's University Press, 2011), 524 p.
}

\section{Simon Jolivet}

Volume 65, numéro 2-3, automne 2011, hiver 2012

URI : https://id.erudit.org/iderudit/1018255ar

DOI : https://doi.org/10.7202/1018255ar

Aller au sommaire du numéro

Éditeur(s)

Institut d'histoire de l'Amérique française

ISSN

0035-2357 (imprimé)

1492-1383 (numérique)

Découvrir la revue

Citer ce compte rendu

Jolivet, S. (2011). Compte rendu de [Olson, Sherry et Patricia Thornton, Peopling the North American City, Montreal 1840-1900 (Montréal et Kingston,

McGill-Queen's University Press, 2011), 524 p.] Revue d'histoire de l'Amérique

française, 65(2-3), 376-379. https://doi.org/10.7202/1018255ar d'utilisation que vous pouvez consulter en ligne.

https://apropos.erudit.org/fr/usagers/politique-dutilisation/ 
Il reste qu'Anik Meunier et Jean-François Piché présentent ici un ouvrage accessible qui rappelle les avancées du monde du travail enseignant et qui permet de faire émerger de manière fort bien illustrée cette importante facette de l'histoire de l'éducation au Québec.

ANDrée Dufour Centre interuniversitaire d'études québécoises - Université Laval Cégep Saint-Jean-sur-Richelieu

Olson, Sherry et Patricia Thornton, Peopling the North American City, Montreal 1840-1900 (Montréal et Kingston, McGill-Queen's University Press, 2011), 524 p.

L'ouvrage est remarquable. Commencé il y a 25 ans, il est issu du travail colossal des géographes-historiennes Sherry Olson et Patricia Thornton. Original dans sa façon de tracer l'évolution et le développement d'une ville et des individus qui l'habitent, l'ouvrage Peopling the North American City, Montreal 1840-1900 marque le couronnement professionnel de deux chercheuses méticuleuses. Il marque aussi un nouveau départ pour l'historiographie québécoise qui, désormais, devra tenir davantage compte des rapports entre le groupe majoritaire franco-catholique et les minorités anglophones.

On pourra bien sûr reprocher le style hermétique, les quelque 130 figures et tableaux (certains superflus) ou encore certains passages rébarbatifs dignes d'une certaine histoire sociale. Il reste, au final, que ce livre est le premier du genre à étudier, sur une aussi longue période, les trois communautés montréalaises les plus influentes du XIX siècle : canadienne-française, catholique irlandaise et protestante anglophone. Regroupant à eux seuls près de $96 \%$ de la population de la ville jusqu'en 1900, ces groupes ont marqué profondément l'histoire du Québec et la culture montréalaise.

Les études sur les anglophones sont encore très rares; la synthèse de Ronald Rudin étant l'exception qui confirme la règle. Qui plus est, l'historiographie a encore peu documenté les relations entre anglophones catholiques/protestants et Canadiens français. Pourtant, les anglophones étaient majoritaires à Montréal pendant un certain temps : «Entre 1831 et 1867 , la majorité de la population est anglophone, atteignant un seuil maximal en 1844, avec 55\%.» (Jean-Claude Robert, Atlas historique de Montréal, Montréal, Libre Expression, 1994, p. 93.) Ce n’est pas par hasard 
si la ville de Montréal se dote en 1833 d'une devise appropriée pour la circonstance: Concordia Salus (le salut par la concorde).

Il est vrai que l'historiographie s'est ouverte à la question de la diversité culturelle depuis deux décennies. Les travaux de Pierre Anctil et de Chantal Ringuet sur la communauté immigrante yiddish à Montréal, ceux de Roberto Perin et de Bruno Ramirez sur les catholiques irlandais ou italiens, ceux de Martin Pâquet et de José Del Pozo, etc., ont montré en quoi l'Autre a enrichi la collectivité québécoise, spécialement en ce qui concerne le $\mathrm{XX}^{\mathrm{e}}$ siècle.

Pourtant, la vie des communautés anglophones du XIX siècle n’a pas fait l'objet d'études aussi importantes. En 1992 déjà, dans un article publié dans la RHAF, Jane Greenlaw notait combien «les chercheurs n'ont pas beaucoup étudié la communauté (anglophone) protestante de Montréal ( Choix pratiques et choix des pratiques : le non-conformisme protestant à Montréal (1825-1842)», 46-1, 1992, p. 93)». Michel Ducharme, dans un excellent ouvrage, en rajoutait : «Si certains groupes ont reçu beaucoup d'attention de la part des historiens... d'autres, telle que l'élite anglophone du Bas-Canada, ont presque été ignorés. " (Le concept de liberté au Canada à l'époque des Révolutions atlantiques (1776-1838), McGill-Queen's University Press, 2010, p. 10).

Le travail de Sherry Olson et Patricia Thornton arrive donc à point. En plus de combler une lacune historiographique importante, les auteures réussissent à saisir la complexité des rapports micro et macro entretenus entre et par les membres de ces trois communautés. Un des exploits de ce livre est de réunir le «collectif » et «l'individuel». Les auteures n'oublient jamais que les trois groupes sont d'abord et fondamentalement constitués d'êtres humains, que ces derniers soient membres des élites ou des ouvriers, des anglophones ou des francophones, des protestants ou des catholiques.

Olson et Thornton offrent une analyse minutieuse des conditions de vie des Montréalais au XIX ${ }^{\mathrm{e}}$ siècle, en rappelant qu'ils sont issus de groupes ethnoculturels distincts et que cela façonne leur rapport au monde. Elles rendent compte d'une réalité souvent négligée dans les travaux en histoire sociale : le fait de devoir tenir compte de la langue, de la religion, de la nationalité et de l'origine ethnique pour bien expliquer les habitudes et les comportements individuels.

Elles affirment que le Montréal du XIX ${ }^{\mathrm{e}}$ siècle est l'œuvre de trois communautés ethnoculturelles distinctes. Le découpage est peut-être sur- 
prenant. Il omet en quelque sorte de parler des Écossais catholiques ou des Écossais protestants, ou encore des Irlando-protestants dont nous ne connaissons encore presque rien. Mais ce découpage est pourtant bien à-propos : les protestants anglophones de Montréal, qu'ils soient irlandais, écossais, anglais ou américains, partagent des affinités religieuses, linguistiques, sociales et culturelles qui les placent dans le haut de l'échelle socioéconomique. Face à la domination et à la richesse des Anglo-protestants (p. 47), les Irlandais catholiques et les Canadiens français sont souvent marginalisés... mais par le fait même facilement repérables.

Comment les Irlandais, les Canadiens français ou les Anglo-Protestants conçoivent-ils collectivement et individuellement leur place à Montréal ? Quelles visions du monde, du quotidien, de l'amour et de la vie entretiennent-ils? Peut-on affirmer qu'ils se rejoignent le plus souvent sur certaines questions fondamentales, malgré les différences religieuses et linguistiques évidentes? Les conclusions d'Olson et Thornton sont claires : non seulement les clivages ethnoculturels sont palpables dès les années 1840 (et notamment au cours des vagues d'immigration irlandaise liées à la Famine), mais ils le sont toujours en 1900. Elles affirment :

Despite continual exchange among the three communities and some intermarriage, their differences persisted over the three or four generations observed, undermining presumptions of the cultural assimilation of migrants (p. 24)

Au-delà de la «structure», il y a donc «la culture». L'étude des communautés ethnoculturelles qui coexistent à Montréal est cruciale pour mieux comprendre la ville et le Québec : «In Montréal, today or yesterday, the cultural cannot be ignored (p. 18).» Olson et Thornton confirment les travaux de Fredrik Barth qui parle de la reproduction des frontières ethniques au fil des générations. S’il est vrai que les Irlandais catholiques réussissent peu à peu à gravir les échelons socio-économiques (et cela probablement plus rapidement que ne le font les Canadiens français), il est pourtant inexact de conclure en l'intégration de ces mêmes Irlandais à la communauté anglo-protestante avant 1900. Cela concorde avec les études récentes sur les Irlandais du Québec, études qui signalent la persistance du sentiment ethnoculturel d'irlandicité au tournant du $\mathrm{XX}^{\mathrm{e}}$ siècle.

Les membres des trois communautés à l'étude évoluent donc en parallèle, se côtoyant au sein d'institutions communes, mais se distinguant aussi dans la manière de vivre le quotidien. Alors que la population de Montréal décuple entre 1840 et 1900, des différences culturelles notables persistent et se reproduisent. En scrutant le parcours de vie d'un 
Montréalais(e) sur 200, les auteures focalisent l'attention sur les événements fondamentaux de tout être humain : sa naissance, ses rapports amoureux, ses recherches d'emplois, ses maladies, sa mort. Elles nous apprennent que le taux de mortalité infantile en très bas âge est deux fois plus important dans les familles canadiennes-françaises qu'anglophones (p. 98). Ou encore que (concernant la notion du célibat) : «... a cultural difference remains, and the Irish stand out, the men three times as likely to be single as French Canadians, the women about twice as likely. Protestant men were more likely to be single than French Canadians, and Protestant women were only a little more likely (p. 167).»

L'ouvrage n'est pas parfait. Le récit n'est pas limpide. La manière de vouloir prouver à tout prix, dans le corps du texte, le sens des démarches méthodologiques rend la lecture difficile. On aurait pu éviter le problème en faisant un meilleur usage des notes et références. Les chapitres abordent des sujets très variés, allant des causes de la mortalité, du taux de nuptialité, du pouvoir d'achat, de la formation de sociétés philanthropiques, de l'allaitement, en passant par l'incidence de la variole et de l'effet des chevaux sur la santé du voisinage. Tous ces sujets sont intéressants, mais, mis ensemble, ils embrouillent parfois l'esprit du lecteur, même spécialisé.

Cet ouvrage remarquable, dans sa forme actuelle, risque d'intéresser au plus haut point les universitaires et ceux qui lisent bien l'anglais. Dommage, parce qu'il est crucial de rendre cet ouvrage accessible à un plus large public, un public qui existe pourtant et qui est avide d'en connaître plus sur l'histoire de Montréal et du Québec. Il est à souhaiter que ce livre majeur soit vite traduit. Il s'agit en effet d'une œuvre très importante pour l'historiographie québécoise.

Simon Jolivet Historien

Pageau, Pierre, Les salles de cinéma au Québec (Québec: Éditions GID, 2009), 414 p.

Professeur de cinéma retraité du cégep Ahuntsic, animateur d'une émission sur le cinéma à Radio centre-ville et rédacteur à la revue Séquences, Pierre Pageau est aussi un grand collectionneur de cartes postales de salles de cinéma. Surtout celles d'avant les années 1960. Voilà qu’au 